\title{
Protective effect of enterovirus-71 (EV71) virus-like particle vaccine against lethal EV71 infection in a neonatal mouse model
}

\author{
LEI CAO $^{1}$, FENGFENG MAO $^{2}$, ZHENG PANG $^{1}$, YAO YI $^{1}$, FENG QIU $^{1}$, \\ RUIGUANG TIAN $^{1}$, QINGLING MENG ${ }^{1}$, ZHIYUAN JIA $^{1}$ and SHENGLI BI ${ }^{1}$ \\ ${ }^{1}$ Department of Viral Hepatitis, National Institute for Viral Disease Control and Prevention, \\ China Center for Disease Control and Prevention, Beijing 102206; ${ }^{2}$ Laboratory Animal Center, \\ The Fourth Military Medical University, Xi'an, Shaanxi 710032, P.R. China
}

Received April 9, 2014; Accepted March 16, 2015

DOI: $10.3892 / \mathrm{mmr} .2015 .3680$

\begin{abstract}
Enterovirus-71 (EV71) is a viral pathogen that causes severe cases of hand, foot and mouth disease (HFMD) among young children, with significant mortality. Effective vaccines against HFMD are urgently required. Several EV71 virus-like particle (VLP) vaccine candidates were found to be protective in the neonatal mouse EV71 challenge model. However, to what extent the VLP vaccine protects susceptible organs against EV71 infection in vivo has remained elusive. In the present study, the comprehensive immunogenicity of a potential EV71 vaccine candidate based on VLPs was evaluated in a neonatal mouse model. Despite lower levels of neutralizing antibodies to EV71 in the sera of VLP-immunized mice compared with those in mice vaccinated with inactivated EV71, the VLP-based vaccine was shown to be able to induce immunoglobulin (Ig)G and IgA memory-associated cellular immune responses to EV71. Of note, the EV71 VLP vaccine candidate was capable of inhibiting viral proliferation in cardiac muscle, skeletal muscle, lung and intestine of immunized mice and provided effective protection against the pathological damage caused by viral attack. In particular, the VLP vaccine was able to inhibit the transportation of EV71 from the central nervous system to the muscle tissue and greatly protected muscle tissue from infection, along with recovery from the viral infection. This led to nearly $100 \%$ immunoprotective efficacy, enabling neonatal mice delivered by VLP-immunized female adult mice to survive and grow with good health. The present study provided valuable additional knowledge of the specific protective efficacy of the EV71 VLP vaccine in vivo, which also indicated that it is a promising potential candidate for being developed into an EV71 vaccine.
\end{abstract}

Correspondence to: Professor Shengli $\mathrm{Bi}$, Department of Viral Hepatitis, National Institute for Viral Disease Control and Prevention, Chinese Center for Disease Control and Prevention, 155 Chang Bai Road, Beijing 102206, P.R. China

E-mail: shengli_bi@163.com

Key words: enterovirus-71, virus-like particle, vaccine, model

\section{Introduction}

Enterovirus 71 (EV71) is a member of the Picornaviridae family of the enterovirus genus and is one of the main etiological agents responsible for hand-foot-mouth disease (HFMD) in humans $(1,2)$. EV71 is a non-enveloped virus with a single-stranded RNA genome consisting of $\mathrm{P} 1, \mathrm{P} 2$ and $\mathrm{P} 3$ regions (3). The P1 protein is further cleaved into VP1, VP3 and $\mathrm{VPO}$ by protease $3 \mathrm{CD}$, while the other two regions encode seven proteins responsible for replication and virulence (3). VP1, VP3 and VP0 can spontaneously co-assemble into the icosahedral empty procapsid (2). A portion of VP0 can be autocleaved to yield VP2 and VP4, which are associated with infectious EV71 virions (4). EV71 infections can cause more severe neurological complications than other enteroviruses and can lead to high morbidity rates in children $(5,6)$. Since its initial identification in 1969, several HFMD epidemics have occurred worldwide, particularly in Asia-Pacific regions $(7,8)$. In China, outbreaks of EV71 infection have been reported throughout the country with increasing prevalence, particularly during the last 10 years (9). Several investigations have focused on the prevention of EV71 infections, and numerous approaches have been tested to develop a safe and effective EV71 vaccine $(10,11)$.

Virus-like particles (VLPs) have attracted increasing attention as great potential vaccine candidates, as they are non-infectious particles consisting of all the major structural proteins, mimicking the organization and conformations of the native particle; however, they are devoid of viral nucleic acids and are non-infectious (12). VLP-based prophylactic vaccines have been successful against hepatitis B virus and human papillomavirus and are now commercially available. Recombinant EV71 VLPs have been shown to be neutralization antibodies and confer a degree of protection from EV71 infection in a neonatal mouse model (13-15).

Variable virus proliferation has been demonstrated in the central nervous system and associated organs during EV71 infections $(16,17)$. Therefore, the generation of immunoprotective responses in infected animals and indicators of pathological responses from the protection by the EV71 vaccine also require an objective assessment. However, to what extent the VLP vaccine protects susceptible organs 
against EV71 infection in vivo remains elusive. Preliminary studies have indicated that neutralizing antibodies induced by VLPs my be able to efficiently neutralize the homologous live EV71 virus and a panel of two C4 strains isolated in China (data not shown). In the present study, the efficacy of an EV71 vaccine candidate based on VLPs was evaluated; furthermore, the significance and value of assessing the immunogenicity and immunoprotection of vaccine candidates in ICR mice were further elucidated by using a range of methods, including pathological, etiological and lethal challenge analyses.

\section{Materials and methods}

Viruses and VLP vaccine preparation. The human EV71 FY-15 strain (C4 genogroup, isolated in Fu Yang, Anhui, China, 2008) was used for immunization. Another highly mouse-adapted virulent EV71 strain (C4 genogroup) supplied by the National Vaccine and Serum Institute (Beijing, China) was used in the challenge experiments. The two EV71 viruses were propagated in rhabdomyosarcoma (RD) cells using minimum essential medium (MEM; Gibco-BRL, Invitrogen Life Technologies, Grand Island, NY, USA) supplemented with $2 \%$ fetal bovine erum (FBS; Gibco-BRL). For virus purification, the FY-15 virus was precipitated with $7 \%$ polyethylene glycol 8000 (Amresco, LLC, Solon, OH, USA) and 2\% $\mathrm{NaCl}$ (Sinopharm Chemical Reagent Co., Ltd., Beijing, China) and then centrifuged $(110,000 \mathrm{xg}, 3 \mathrm{~h})$ over $15 \%$ cesium chloride (CsCl; Sinopharm Chemical Reagent Co., Ltd.). Virus pellets were re-suspended in PBS ( $\mathrm{pH} 7.4)$, sonicated for $30 \mathrm{sec}$ and centrifuged $(10,0000 \mathrm{xg}, 20 \mathrm{~h})$ over a continuous $\mathrm{CsCl}$ gradient (10-40\%). The resultant virus bands were dialyzed against phosphate-buffered saline (PBS). Purified FY-15 virus was examined by eryo-electron microscopy (Cryo-EM; Tecnai Tf20; FEI, Houston, TX, USA) and inactivated with 1/4,000 formalin (Sigma-Aldrich, St Louis, MO, USA) for $96 \mathrm{~h}$ at $37^{\circ} \mathrm{C}$ (18). Another mouse-adapted EV71 virus was harvested and centrifuged $(5,000 \mathrm{xg}, 10 \mathrm{~min})$ to remove debris. The viral titer was evaluated using RD cells by microtitration assay and expressed as the 50\% tissue culture-infective dose $\left(\mathrm{TCID}_{50}\right)$ (19).

EV71 VLPs were produced in serum-free medium as previously described (20). Briefly, Sf9 cells (Invitrogen Life Technologies) were infected with a recombinant baculovirus (AcMNPV-P1-3CD; constructed previously in the Department of Viral Hepatitis) for co-expression of P1 and 3CD derived from the EV71 FY-15 strain at a multiplicity of infection (MOI) of 1 in 11 CellSTACK-10 culture chambers (Corning-Costar, Corning, NY, USA) at $27^{\circ} \mathrm{C}$ for $96 \mathrm{~h}$. The infected cells were harvested with a Tris- $\mathrm{NaCl}$ (Invitrogen Life Technologies) buffer containing 1\% NP-40 (Sigma-Aldrich) and cell debris was removed by centrifugation (9,000 xg, $20 \mathrm{~min})$. Then the supernatant was centrifuged over $15 \% \mathrm{CsCl}$ and a discontinuous sucrose gradient (10-60\%). Purified VLPs were examined by transmission electron microscopy (TEM; Tecnai 12; FEI). The protein concentrations were quantified using a Nanodrop (Thermo Fisher Scientific, Waltham, MA, USA). A solution of the inactivated virus and VLPs each was then mixed with adjuvant $\mathrm{Al}(\mathrm{OH})_{3}(2 \mathrm{mg} / \mathrm{ml}$; Sinovac Biotech Co., Ltd, Beijing, China) at a volumetric ratio of 1:1 for in vivo studies.
Immunization of adult mice and sampling procedures. Three groups ( $n=10 /$ group) of 4-5 week-old female ICR mice (Beijing HFK Bioscience Co., Ltd., Beijing, China) were immunized by intramuscular injection with $5 \mu \mathrm{g}$ (100 $\mu \mathrm{l} /$ mouse) of either purified VLPs, inactivated whole virus or PBS. The mice were subjected to a $12 \mathrm{~h}$ dark $/ 12 \mathrm{~h}$ light cycle, with ad libitum access to food and water. The mice were housed 5 mice/cage in a room maintained at $19-24^{\circ} \mathrm{C}$ with $47-63 \%$ relative humidity. Animal treatment and care were provided in accordance with the guidelines of the National Institute for Viral Disease Control and Prevention, China Center for Disease Control and Prevention (Beijing, China). All procedures used in the present study were approved by the Animal Care and Welfare Committee at the National Institute for Viral Disease Control and Prevention, China Center for Disease Control and Prevention. The immunization schedule consisted of three inoculations administered at two-week intervals. Blood was sampled from the tail at weeks $0,2,4,6,8,10$ and 14 for monitoring the immune responses. Mice ( 3 or 4 per group) were sacrificed at week 6 and 10 for measurement of the cellular immune responses. Lung wash, vaginal wash and small intestine samples were collected at week 6 in order to test secretory immunoglobulin (Ig)A levels. All these samples were immediately placed on ice and stored with $100 \mathrm{mM}$ phenylmethyl sulfonyl fluoride (Sigma-Aldrich) at $-20^{\circ} \mathrm{C}$ until further analysis. The remaining mice in each group were used to breed neonatal mice for lethal virus challenge experiments at week eight (week four post-boost) and sacrificed at week 14.

Measurement of EV71-specific antibody. Anti-EV71 IgG and $\mathrm{IgG}$ subclasses in serum as well as $\operatorname{IgA}$ in mucosal secretions were measured by ELISA. In brief, plates coated with inactivated native virus $(5 \mu \mathrm{g} / \mathrm{ml})$ were blocked (PBS containing $0.05 \%$ Tween 20 (Sinopharm Chemical Reagent Co., Ltd.) and 5\% bovine serum albumin (Amresco, LLC) and washed prior to the addition of the two-fold serially diluted serum samples $\left(1: 2^{2}-22^{2}\right)$ and 1:2-diluted mucosal secretion samples were added. Plates were incubated for $1 \mathrm{~h}$ and washed prior to addition of $0.15 \mu \mathrm{g} / \mathrm{ml}$ horseradish-peroxidase (HRP)-conjugated goat anti-mouse IgG (1:30,000 dilution; cat. no. A9917; Sigma-Aldrich) or $0.125 \mu \mathrm{g} / \mathrm{ml}$ goat anti-mouse IgA (HRP) secondary antibody (1:8,000 dilution; cat. no. ab97235; Abcam, Cambridge, MA, USA) was added. The anti-EV71 IgG isotypes in mouse sera were determined using a mouse antibody isotyping kit (ISO 2; Sigma-Aldrich) according to the manufacturer's instructions. After 30 minutes, color development was initiated by adding $100 \mu \mathrm{l}$ tetramethylbenzidine substrate (Sigma-Aldrich) and terminated by adding $50 \mu \mathrm{l}$ of $\mathrm{H}_{2} \mathrm{SO}_{4}$ (2M; Sinopharm Chemical Reagent Co., Ltd.). The optical density at $40 \mathrm{~nm}\left(\mathrm{OD}_{450}\right)$ was read using an ELISA reader (FI-01621; Thermo Scientific Multiskan ${ }^{\mathrm{TM}}$ GO; Thermo Fisher Scientific Oy, Vantaa, Finland).

Neutralizing antibody assays. Microneutralization assays were performed against EV71 in RD cells. Briefly, $50 \mu 1$ two-fold diluted serum was mixed with $50 \mu 1100$ TCID $_{50}$ EV71 in 96-well plates. RD cells were added after $1 \mathrm{~h}$ and incubated for $3-4$ days at $37^{\circ} \mathrm{C}$. The neutralizing titer was determined as the highest dilution that gave no cellular cytopathic effects (CPE). 
A

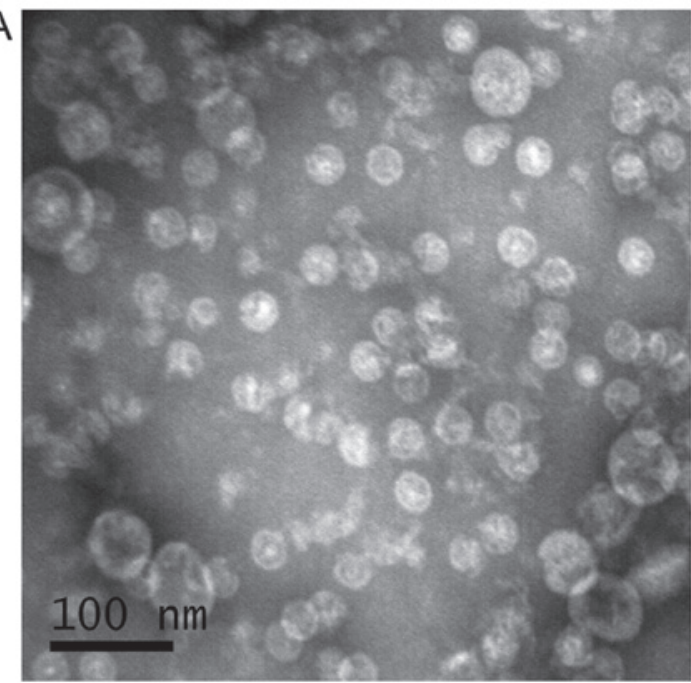

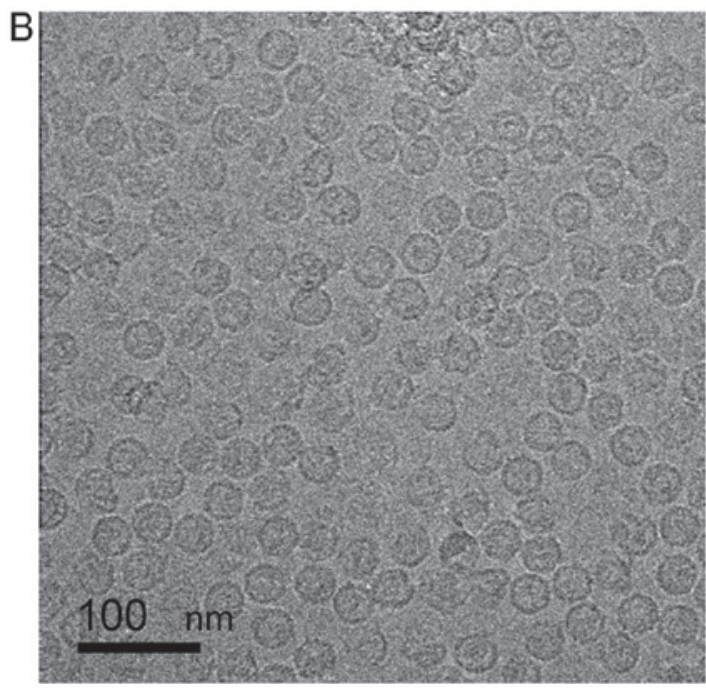

Figure 1. Characterization of purified VLPs. (A) Transmission electron microscopy image of VLPs produced by recombinant baculovirus (AcMNPV-P1-3CD)-infected Sf9 cells co-expressing EV71 P1 and 3CD. VLPs were purified by ultracentrifugation. (B) EV71 virions after ultracentrifugation were characterized by cryo-electron microscopy. Scale bar, $100 \mathrm{~nm}$. EV, enterovirus; VLP, virus-like particle.

Enzyme-linked immunospot (ELISpot) assays. interleukin (IL)-4-,interferon (IFN)- $\gamma$ - and IgA-secreting splenocytes were measured using ELISpot for mouse IL-4, IFN- $\gamma$ (U-Cytech, Utrecht, the Netherlands) and IgA kits (Mabtech AB, Nacka Strand, Sweden). Briefly, for detection of virus-specific IL-4 and IFN- $\gamma$, 96-well microplates (Millipore, Billerica, MA, USA) were pre-coated with anti-IL- 4 or anti-IFN- $\gamma$ monoclonal antibody $(\mathrm{mAb})$ and incubated overnight at $4^{\circ} \mathrm{C}$. Plates were blocked with RPMI-1640/10\% FBS for $1 \mathrm{~h}$ at room temperature, followed by addition of splenocytes (3x10 $/$ well). Subsequently, $250 \mu \mathrm{g} / \mathrm{ml}$ inactivated EV71 virus was added (20 $\mu \mathrm{l} /$ well) to stimulate effector cells. Phytohaemagglutinin (PHA; $9 \mu \mathrm{g} /$ well, $37^{\circ} \mathrm{C}, 16 \mathrm{~h}$; Gibco-BRL) stimulation was used for the positive control. For IgA ELISpot, microplates were first coated overnight with $10 \mu \mathrm{g} / \mathrm{ml}$ anti-IgA antibody or $30 \mu \mathrm{g} / \mathrm{ml}$ inactivated EV71 virions and blocked with PBS-10\% FBS for $30 \mathrm{~min}$. Then splenocytes (1x105/well) were added. After incubation of the splenocytes for 16-18 h, following removal of the cells, plates were processed according to the manufacturer's instructions. Spot-forming cells (SFC) were counted using an automated ELISpot reader (Cellular Technology Ltd, Shaker Heights, OH, USA).

Lethal viral challenges in neonatal mice. Four weeks after the final immunization, female adult mice were allowed to mate. The neonatal mice were challenged intracerebrally with the EV71 virus attack strain $\left[10^{6}\right.$ PFUs (plaque-forming units) $/ \mathrm{ml}$, $10 \mu \mathrm{l} /$ mouse] within $72 \mathrm{~h}$ following birth. Mice were monitored for mortality daily over 14 days. In addition, three neonatal mice from each group were used for histopathological analysis at five days after EV71 challenge. The neonatal mice were sacrificed using isoflurane (Sigma-Aldrich). Extracted organs were fixed in $4 \%$ paraformaldehyde for $24 \mathrm{~h}$ and processed for paraffin embedding. Tissues sections $(5 \mu \mathrm{m})$ were deparaffinized using xylene (Sinopharm Chemical Reagent Co., Ltd.) and hydrated with a graded series of alcohol (Sinopharm Chemical Reagent Co., Ltd.). Sections were stained with hematoxylin and eosin (Sigma-Aldrich), for morphological evaluation. Immunohistochemical analyses were performed as described previously (21). Briefly, hydrated sections were treated with $0.25 \%$ trypsin (Invitrogen Life Technologies) solution containing $0.5 \% \mathrm{CaCl}_{2}$ (Sinopharm Chemical Reagent Co., Ltd.) in PBS for $30 \mathrm{~min}$ and incubated with $3 \% \mathrm{H}_{2} \mathrm{O}_{2}$ (Sinopharm Chemical Reagent Co., Ltd,) in methanol to block endogenous peroxidase activity, followed by incubation with PBS containing 5\% BSA. The treated sections were then incubated with anti-EV71 mAb (1:300 dilution; cat, no. 05-0001; AbMax Biotechnology Co., Ltd., Beijing, China) at $4^{\circ} \mathrm{C}$ overnight. The sections were washed three times with PBS and then incubated with HRP-conjugated goat anti-mouse IgG (1:1,000 dilution; cat. no. ZB-2305; ZSGB-BIO, Beijing, China) for $1 \mathrm{~h}$ at $37^{\circ} \mathrm{C}$. The sections were visualized using 3-3'diaminobenzidine (10 $\mathrm{min})$ and hematoxylin (30 sec), then examined using a light microscope (IX51; Olympus Corporation, Tokyo, Japan).

Statistical analysis. All data obtained were processed and analyzed using SPSS software (version 16.0; SPSS, Inc., Chicago, IL, USA). Figures were plotted and analyzed using GraphPad Prism software (GraphPad, La Jolla, CA, USA). $\mathrm{P}<0.05$ was considered to indicate a statistically significant difference between values.

\section{Results}

Characterization of purified VLPS. Purified VLPs and EV71 virus were visualized by TEM and Cryo-EM. Purified VLPs (Fig. 1A) exhibited icosahedral morphology similar to those of EV71 virions (Fig. 1B) and the size was $\sim 25-27 \mathrm{~nm}$. These EV71 VLPs were used as a vaccine in the subsequent experiments.

Humoral responses elicited by EV71 VLP immunization. Neutralization titration of sera of the mice showed that compared with levels in the control group, high levels of anti-EV71 IgG antibodies in the serum of mice were present at 2 weeks post-boost immunization with purified VLPs, 
Table I. IgG subtype profile in mouse sera ${ }^{\mathrm{a}}$.

$\mathrm{OD}_{450}$ of ELISA for EV71 specific IgG at serum dilution of 1:100

\begin{tabular}{lcccc}
\cline { 2 - 5 } Type of antigen & IgG1 & IgG2a & IgG2b & IgG3 \\
\hline VLPs $(\mathrm{n}=10)$ & $1.644(0.111)$ & $0.370(0.059)$ & $0.884(0.042)$ & $0.248(0.085)$ \\
Inactivated EV71 $(\mathrm{n}=10)$ & $1.770(0.154)$ & $0.958(0.082)$ & $1.620(0.106)$ & $0.759(0.054)$ \\
PBS $(\mathrm{n}=10)$ & $0.111(0.011)$ & $0.123(0.014)$ & $0.114(0.004)$ & $0.138(0.022)$
\end{tabular}

${ }^{a}$ Three groups of mice were immunized with EV71 VLPs, inactivated EV71 or PBS. Six weeks after the first immunization, mouse sera were collected and assayed for specific anti-EV71 IgG subtypes by ELISA. Serum samples of each vaccinated group were pooled and tested in triplicate in three separate experiments. The relative titer is expressed as the $\mathrm{OD}_{450}$ with the standard deviation shown in parentheses. $\mathrm{EV}$, enterovirus; VLP, virus-like particle; PBS, phosphate-buffered saline; $\mathrm{OD}_{450}$, optical density at $450 \mathrm{~nm}$; IgG, immunoglobulin G.

A

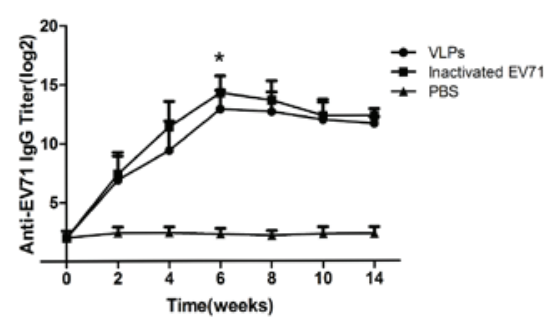

C

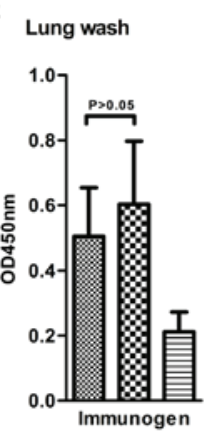

F IFN-Y

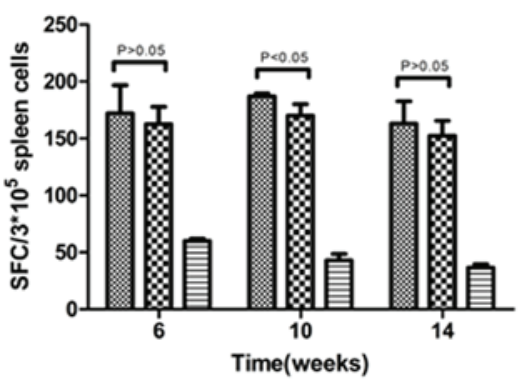

B

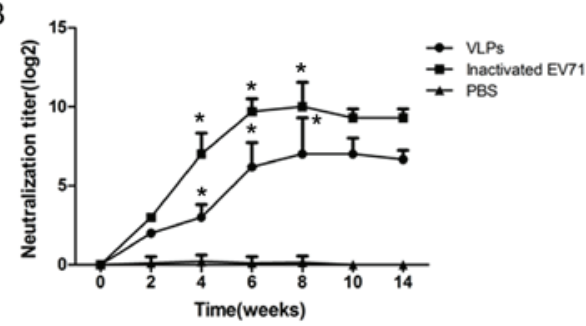

E Intestinal wash

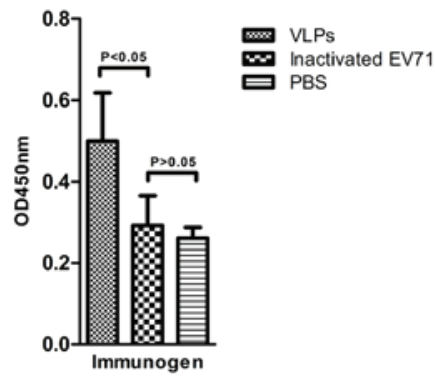

D Vaginal wash

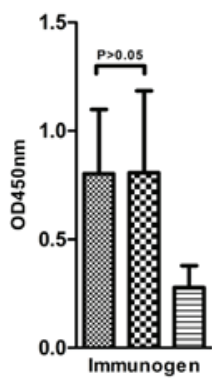

G IL-4

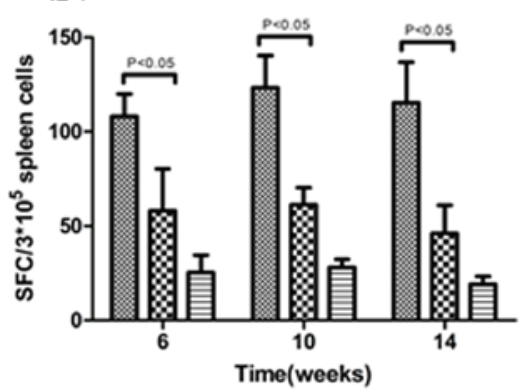

$\mathrm{H}$

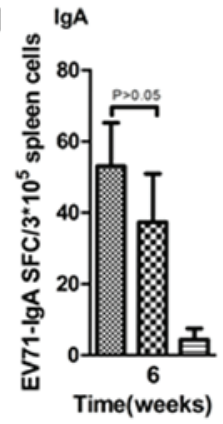

$\infty$ VLPS Inactivated EV71 DPBS

Figure 2. Titer profiles of humoral and cellular immune responses to EV71 in mouse models. The geometric mean titers $(\log 2)$ of (A) total IgG, (B) neutralizing antibody to EV71 and (C-E) anti-EV71 secretory IgA secreted at mucosal surfaces were determined using ELISA (for total anti-EV71 IgG titration, the positive cut-off OD value was defined as 2.1 times that of normal mouse serum) and neutralization techniques. Enzyme-linked immunospot detection of specific responses of (F) IFN- $\gamma$-secreting T cells, (G) IL-4-secreting T cells or (H) IgA-secreting spleen cells to EV71 in spleen lymphocytes from immunized mice. All values are expressed as the mean \pm standard deviation from 3 or 4 mice per group. EV, enterovirus; VLP, virus-like particle; SFC, spot-forming cells, PBS, phosphate-buffered saline; $\mathrm{OD}_{450}$, optical density at $450 \mathrm{~nm}$; IFN, interferon; Ig, immunoglobulin.

reaching a maximum of $2^{12}$ (Fig. 2A). Compared with the VLPs, inactivated EV71 induced slightly higher levels of anti-EV71 antibodies with a significant difference at week six $(\mathrm{P}<0.05)$. In the two groups, the time-dependent changes in antibody levels and magnitudes of the response were similar. Peak levels of antibodies following the two types of antigen immunization were detected at week 6 and maintained for at least 14 weeks. The inactivated EV71-immunized group 

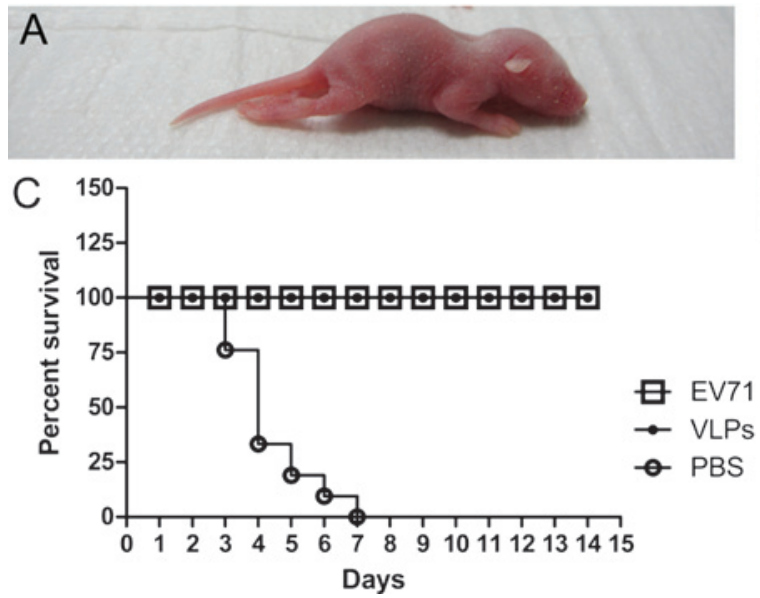

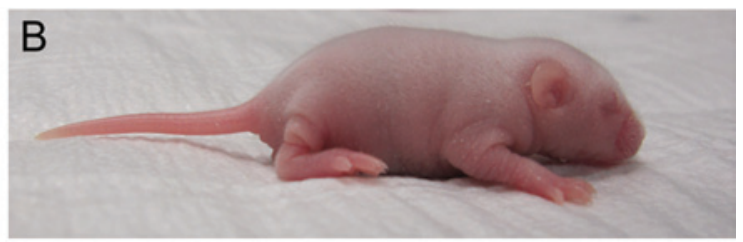

Figure 3. Viral infection of neonatal ICR mice with a lethal EV71 dose ( $10^{6}$ plaque-forming units per mouse). Representative images showing symptoms of (A) unimmunized and (B) VLP-immunized mice after intracerebral challenge at day three post-infection. Symptoms included weight loss, hunched back, reduced motility, hind limb paralysis and eventual mortality of unimmunized mice. VLP-immunized mice survived. (C) Survival of immunized and unimmunized mice after intranasal challenge with lethal doses of EV71. ( $\mathrm{n}=20$ for each group). EV, enterovirus; VLP, virus-like particle; PBS, phosphate-buffered saline.

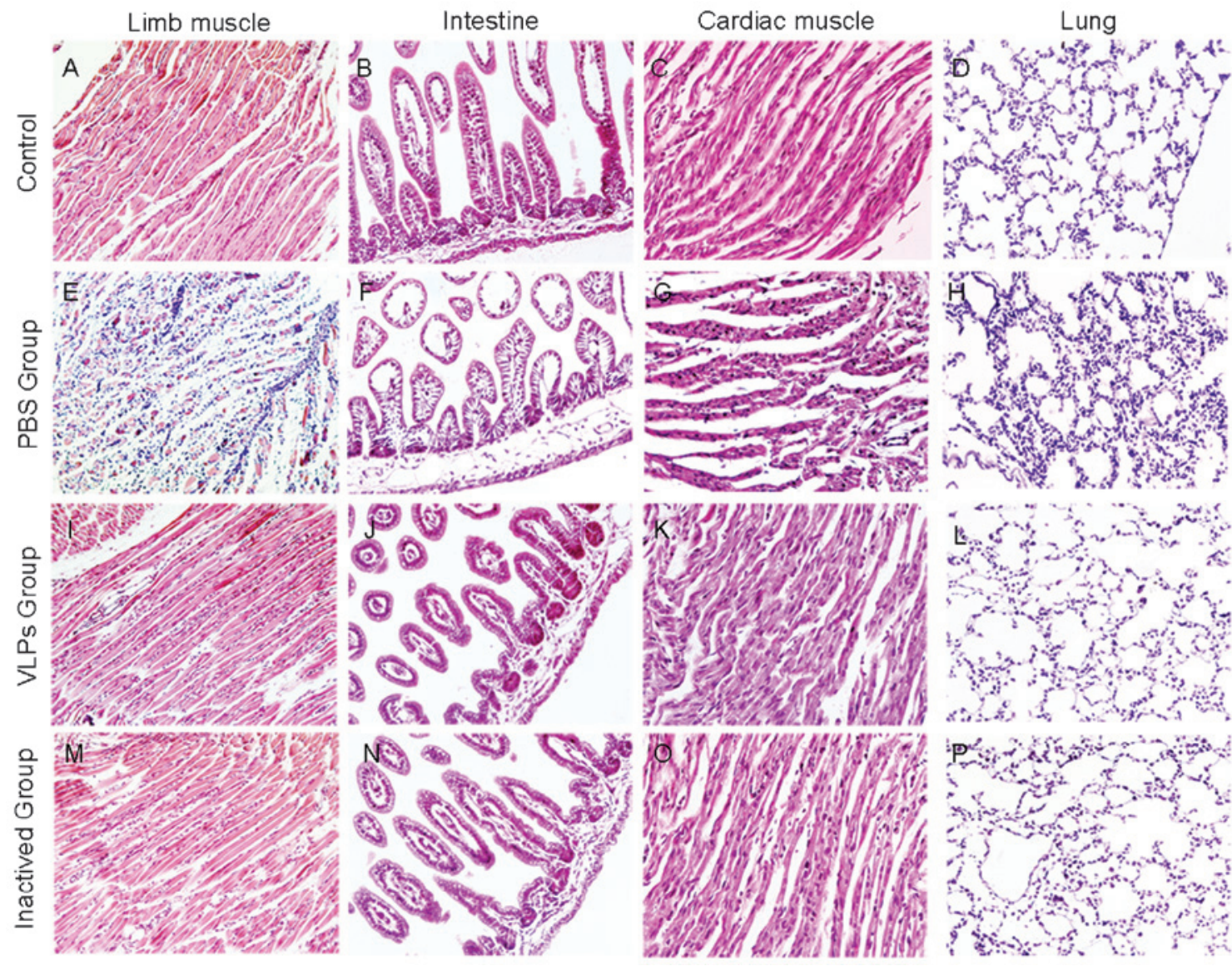

Figure 4. Pathological changes in various tissues of mice infected with enterovirus 71 by intracerebral inoculation. Histopathological examinations were performed on 5- $\mu \mathrm{m}$ sections of paraffin-embedded tissues or organs stained with hematoxylin and eosin. Magnification, x200. VLP, virus-like particle; PBS, phosphate-buffered saline.

exhibited higher levels of all IgG subtypes (Table I); however, high levels of IgG1 and IgG2b and low levels of IgG2a and IgG3 were also detected in the serum of the VLP-immunized group, indicating a mixed $\mathrm{Th} 1 / \mathrm{Th} 2$ response. The capacity of antibodies generated by VLP immunization to neutralize the homologous live EV71 virus was investigated (Fig. 2B).
In VLP-immunized mice, significantly increased neutralization titers were observed post-boost compared with those pre-vaccination (week 4-8) $(\mathrm{P}<0.05)$; however, these were lower than those in the formalin-inactivated EV71-immunized mice $(\mathrm{P}<0.05)$. While the neutralization titer profile for the group immunized with VLPs was similar to that for the group 

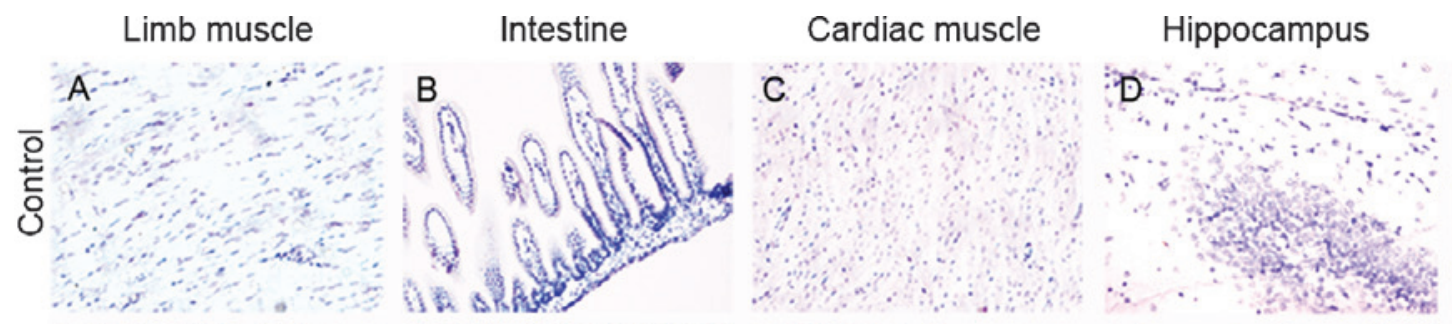

\section{Cerebral cortex}
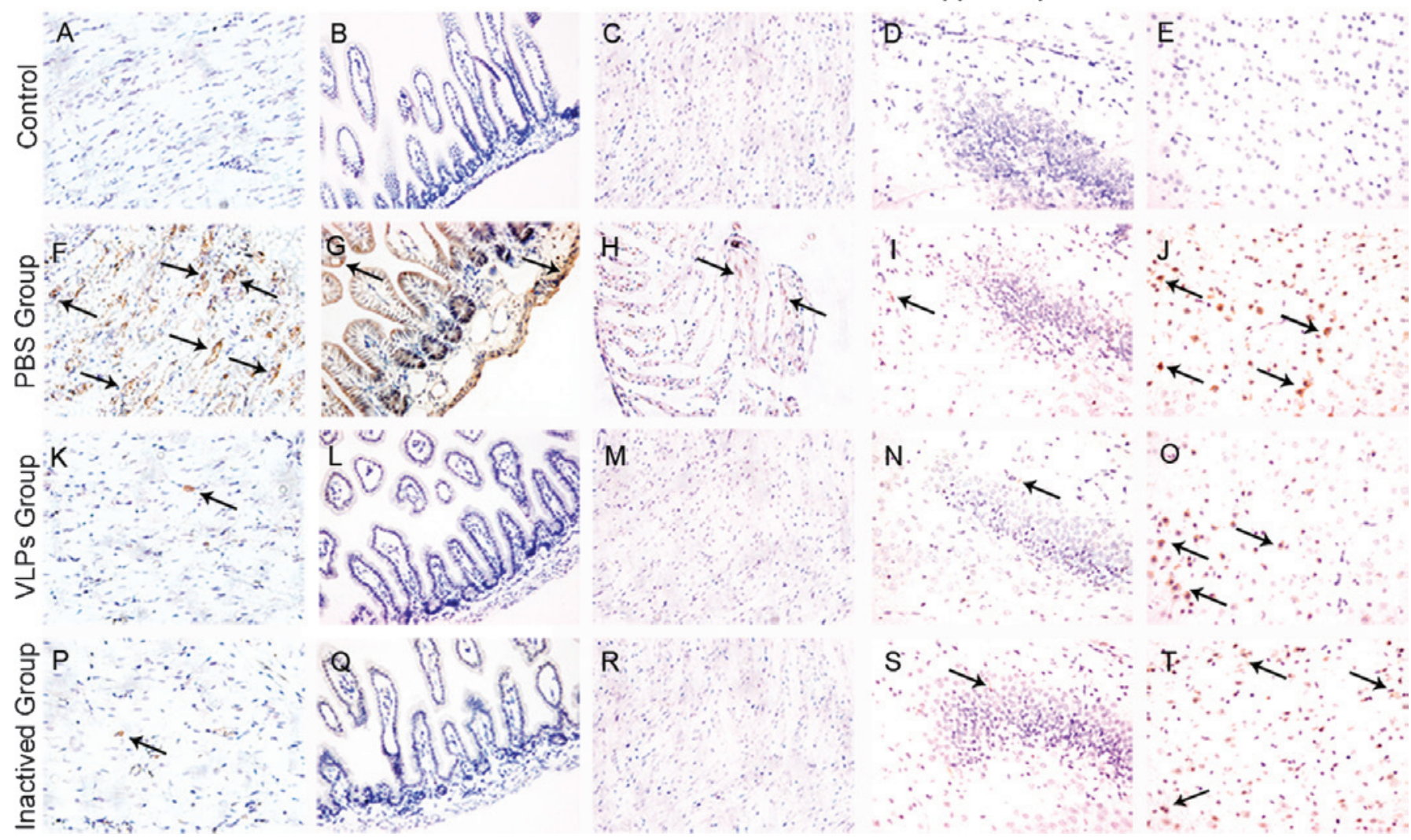

Figure 5. Immunohistochemical analysis of various tissues from mice infected with EV71 by intracerebral inoculation. Sections (5 $\mu$ m) of paraffin-embedded tissues or organs were prepared and EV71 was detected with mouse anti-EV71 monoclonal antibody and visualized with diaminobenzidine substrate. Arrows indicate positivity for viral anitigens; magnification, x200. EV, enterovirus; VLP, virus-like particle; PBS, phosphate-buffered saline.

immunized with inactivated EV71, neutralization titers in the two groups were greatest at week 8 and persisted for at least 14 weeks.

The specific $\operatorname{Ig}$ A levels in the lung, vaginal and intestinal secretions from the vaccinated mice after the third immunization with EV71 VLPs showed a significant difference from those in the PBS group $(\mathrm{P}<0.05$; Fig. $2 \mathrm{C}-\mathrm{E})$. However, the specific IgA responses in the intestinal samples from inactivated EV71-immunized mice were not significantly different from those in the PBS group, which may be due to incomplete homogenization processing, complex composition and proteases in the intestinal tract.

Cellular immune responses elicited by VLP immunization. Compared with splenocytes from mice immunized with inactivated EV71, splenocytes from VLP-immunized mice produced the same levels of EV71-specific IFN- $\gamma$-producing T-cells ( $\mathrm{P}>0.05$, Fig. 2F) and higher levels of IL-4-producing T-cells in response to EV71 antigens $(\mathrm{P}<0.05$; Fig. 2G), confirming the successful elicitation of Th1 and Th2 cellular immune responses. The EV71-specific IgA-secreting splenocytes were also quantified at week 6 . Increased numbers of IgA-memory cells in mouse splenocytes in the VLP group and the inactivated EV71 group were found following immunization compared with those in the PBS control group (Fig. 2H; $\mathrm{P}>0.05)$. These results suggested that vaccination with EV71 VLPs leads to the generation of IgA-memory splenocytes with the capacity to recognize EV71 virions.
Challenge with EV71 results in mortality of neonatal mice, which can be prevented by vaccination of their mothers. To assess the efficacy of passive protection against the homologous EV71, neonatal ICR mice were challenged intracerebrally with EV71. As the result, nearly all inoculated mice from the PBS control group displayed a range of clinical symptoms (weight loss, hunched back, reduced motility, ruffled coat and hind limb paralysis) over the course of infection followed by death from day three (Fig. 3A and C). By contrast, all of the neonatal mice delivered by VLP-immunized and inactivated EV71 immunized female adult mice were able to survive, grew normally and were of good health, and the immunoprotective efficacy of the vaccines reached nearly $100 \%$ (Fig. 3B). These results demonstrated that VLP immunization conferred passive protection that was passed on from the female parental mice to the neonatal mice.

Histopathological analysis shows that vaccination protects major organs of neonatal mice challenged with EV71. The pathological changes in the brain and other major organs of immunized and non-immunized control neonatal mice were examined in parallel (Fig. 4). Compared with normal control mice, certain pathological changes were present in the PBS group (Fig. 4A-H). The hind limb muscle was most severely affected by severe diffuse necrotizing myositis and minor lymphocytic infiltration, which is likely to be responsible for the observed hind limb paralysis (Fig. 4E). The intestines revealed dilation of the villi and mild villous blunting with 
associated crypt hyperplasia, suggesting substantial tissue damage in the small intestines (Fig. 4F). A pathological response comprising minor myocardial interstitial edema and occasional scattered mononuclear cells invading cardiac muscles were also observed (Fig. 4G). Prominent pathological manifestations of pulmonary inflammation in lung tissue included a widened alveolar wall and extensive inflammatory cell aggregation (Fig. 4H). By contrast, under the protection of the VLP vaccines, only mild muscle atrophy was observed in the limb musculature (Fig. 4I and M). No other pathological changes were noted.(Fig. 4J-L and N-P). In addition, the limb musculature of the neonatal mice immunized with VLPs or inactivated EV71 displayed slight myofiber regeneration within nuclear rowing, suggesting signs of recovery from the viral infection.

Further immunohistochemical analyses were in accordance with the results of the pathological analysis (Fig. 5). EV71 infiltration was mainly observed in the hind limb muscles and to a certain extent in the small intestines and cardiac muscles in the PBS group (Fig. 5F-H). Low amounts of EV71 were detected in the hippocampus, while a high level of EV71 infiltration was detected in the cerebral cortex in all groups, which may be due to the intracerebral injection of the EV71 virus attack strain in the lethal EV71 challenge experiment (Fig. 5I and J). Following vaccine-mediated immunization, only very low levels of EV71 antigen were detected in the hind limb muscles (Fig. 5K-M and P-R). All these observations demonstrated the marked immunoprotective efficacy of EV71 VLP immunization against EV71 infection.

\section{Discussion}

Preliminary studies have indicated that neutralizing antibodies induced by VLPs were able to efficiently neutralize the homologous live EV71 virus and a panel of two C4 strains isolated in China (data not shown). Although VLPs induced similar levels of anti-EV71 IgG compared with inactivated EV71, the neutralization titers in the former case were lower. VP1 is a major antigenic epitope and has an important role in neutralizing the EV71 virus $(22,23)$. According to studies by in Lin et al (11) and Wang et al (24), residues 210-220 of VP1, which form part of an important neutralizing epitope of EV71, lie on the capsid surface, alongside the VP2 EF loop (residues 136-150), to form a single epitope. Disruption in the order of residues 211-217 upon particle expansion may account for the reduced immunogenicity $(11,24)$. The VP1 in the VLPs used in the present study was hypothesized to be similar with the VP1 in the empty EV71 virus particles (sedimentation, $80 \mathrm{~S}$; 60 copies of VP0, VP1 and VP3) cultivated and separated by Wang et al (24). Slight conformational changes of VP1 in empty particles may affect their neutralizing capacity.

Although antibody responses are critical for protection against EV71 infection, certain studies suggested that cellular immune responses correlate with the clinical severity of HFMD and have an important role in human immunity $(13,15)$. Chang et al (13) suggested that lower EV71-specific cellular responses may be associated with immunopathogenesis of EV71-associated pulmonary edema. The different Th1 and Th2 immune response profiles corresponded to the activation of two distinct major sub-sets of $\mathrm{T}$ cells characterized by their pattern of cytokine production (25). The present study indicated that VLP vaccine immunization generated good IFN- $\gamma$ responses and even better IL-4 responses to the EV71 antigen in mice. The ELISpot assay further demonstrated the presence of EV71-specific IgG- and IgA-secreting cells in spleens of the VLP-vaccinated mice, but not of the PBS-immunized mice. The IgG subtype profile showed that the EV71 VLP vaccine induced high levels of IgG1 and IgG2b, thus confirming the generation of a mixed Th1 and Th2 response. These results were consistent with those obtained following influenza virus and human papilloma virus VLP-immunization $(26,27)$. The production of IgA-memory spleen cells detected following VLP vaccine immunization further confirmed successful priming by VLPs.

Protection against viral infectivity in animal models is regarded as the optimal test of vaccine efficacy (23). EV71 was presumed to infect the central nervous system (CNS) via peripheral nerves in muscle tissue followed by active retrograde axonal transport to the CNS (28). It was also reported that muscle cells were the main site of proliferation of the EV71 virus strain found around Fuyang in China $(29,30)$. Additional studies demonstrated that skeletal muscle was the main tissue supporting EV71 replication in mice. In addition to CNS injury, necrotic myositis may also be responsible for the paralysis and death observed in EV71-infected mice (31). Thus, protection against further infection of the CNS or skeletal muscle by preventing EV71 from attacking muscle tissue and neurons is particularly important. Based on the abovementioned studies, the present study performed further pathological and etiological analyses on different organs to understand the specific passive immunoprotective effects in vivo. It was found that the VLP vaccine was able to inhibit the transport of EV71 from the CNS to muscle tissue and prevent infection of muscle tissue along with recovery from the viral infection. The prevention of pathological injury by effective resistance to EV71 infection in intestine and cardiac muscle tissues mediated by VLP vaccine immunization is also of critical importance. This passive protection offered great immunoprotective efficacy from the VLP or inactivated EV71 vaccines, reaching nearly $100 \%$. Of note, the results indicated that VLP vaccines not only prevented the virus from causing serious damage to the body, but also promoted a quick recovery of the body from minor injuries resulting from EV71 infection.

It is likely that these results not only confirmed the immunoprotective effect of the experimental VLP vaccine against EV71, but also provided valuable additional insight to aid in further exploring the detailed mechanism of action of the vaccine, and supported the protective role for the VLP vaccine in resistance and resolution following EV71 infection.

\section{Acknowledgements}

The authors would like to thank Dr Wuling Xie and Dr Baoyun Zhang (National Institute for Viral Disease Control and Prevention, Beijing, China) for technical assistance in tissue paraffin embedding, paraffin sectioning and immunohistochemical analysis. The authors also thank Dr Le Sun (AbMax Biotechnology Co., Ltd, Beijing, China) for critical reading of the manuscript. The present study was supported by a grant from the National Key Technology Research and Development 
Program of the Ministry of Science and Technology of China (no. 2008BAI69B02).

\section{References}

1. Chan KP, Goh KT, Chong CY, Teo ES, Lau G and Ling AE: Epidemic hand, foot and mouth disease caused by human enterovirus 71, Singapore. Emerg Infect Dis 9: 78-85, 2003.

2. McMinn PC: An overview of the evolution of enterovirus 71 and its clinical and public health significance. FEMS Microbiol Rev 26: 91-107, 2002.

3. Brown BA and Pallansch MA: Complete nucleotide sequence of enterovirus 71 is distinct from poliovirus. Virus Res 39: 195-205, 1995.

4. Liu CC, Chou AH, Lien SP, Lin HY, Liu SJ, Chang JY, Guo MS, Chow YH, Yang WS, Chang KH, et al: Identification and characterization of a cross-neutralization epitope of enterovirus 71. Vaccine 29: 4362-4372, 2011.

5. Qiu J: Enterovirus 71 infection: A new threat to global public health? Lancet Neurol 7: 868-869, 2008.

6. Wang SM, Lei HY, Huang KJ, Wu JM, Wang JR, Yu CK, Su IJ and Liu CC: Pathogenesis of enterovirus 71 brainstem encephalitis in pediatric patients: Roles of cytokines and cellular immune activation in patients with pulmonary edema. J Infect Dis 188: 564-570, 2003.

7. Cardosa MJ, Perera D, Brown BA, Cheon D, Chan HM, Chan KP, Cho $\mathrm{H}$ and McMinn P: Molecular epidemiology of human enterovirus 71 strains and recent outbreaks in the Asia-Pacific region: Comparative analysis of the VP1 and VP4 genes. Emerg Infect Dis 9: 461-468, 2003.

8. Fujimoto T, Chikahira M, Yoshida S, Ebira H, Hasegawa A, Totsuka A and Nishio O: Outbreak of central nervous system disease associated with hand, foot, and mouth disease in Japan during the summer of 2000: Detection and molecular epidemiology of enterovirus 71. Microbiol Immunol 46: 621-627, 2002.

9. Yi L, Lu J, Kung HF and He ML: The virology and developments toward control of human enterovirus 71. Crit Rev Microbiol 37: 313-327, 2011.

10. Chong P, Hsieh SY, Liu CC, Chou AH, Chang JY, Wu SC, Liu SJ, Chow YH, Su IJ and Klein M: Production of EV71 vaccine candidates. Hum Vaccin Immunother 8: 1775-1783, 2012.

11. Lin YL, Yu CI, Hu YC, Tsai TJ, Kuo YC, Chi WK, Lin AN and Chiang BL: Enterovirus type 71 neutralizing antibodies in the serum of macaque monkeys immunized with EV71 virus-like particles. Vaccine 30: 1305-1312, 2012.

12. Noad R and Roy P: Virus-like particles as immunogens. Trends Microbiol 11: 438-444, 2003.

13. Chang LY, Hsiung CA, Lu CY, Lin TY, Huang FY, Lai YH, Chiang YP, Chiang BL, Lee CY and Huang LM: Status of cellular rather than humoral immunity is correlated with clinical outcome of enterovirus 71. Pediatr Res 60: 466-471, 2006.

14. Ku Z, Ye X, Huang X, Cai Y, Liu Q, Li Y, Su Z and Huang Z: Neutralizing antibodies induced by recombinant virus-like particles of enterovirus 71 genotype $\mathrm{C} 4$ inhibit infection at preand post-attachment steps. PLoS One 8: e57601, 2013.

15. Chang LY, Chang IS, Chen WJ, Huang YC, Chen GW, Shih SR, Juang JL, Shih HM, Hsiung CA, Lin TY, et al: HLA-A33 is associated with susceptibility to enterovirus 71 infection. Pediatrics 122: 1271-1276, 2008.
16. Khong WX, Foo DG, Trasti SL, Tan EL and Alonso S: Sustained high levels of interleukin-6 contribute to the pathogenesis of enterovirus 71 in a neonate mouse model. J Virol 85: 3067-3076, 2011.

17. Zhang Y, Cui W, Liu L, Wang J, Zhao H, Liao Y, Na R, Dong C, Wang L, Xie Z, et al: Pathogenesis study of enterovirus 71 infection in rhesus monkeys. Lab Invest 91: 1337-1350, 2011.

18. Ong KC, Devi S, Cardosa MJ and Wong KT: Formaldehyde-inactivated whole-virus vaccine protects a murine model of enterovirus 71 encephalomyelitis against disease. J Virol 84: 661-665, 2010.

19. Hsiung GD: Virus assay, neutralization test and antiviral assay. In: Hsiung's Diagnostic Virology. 4th ed. Hsiung GD, Fong CKY and Landry ML (eds). Yale University Press, New Haven, pp46-55, 1994.

20. Cao L, Yi Y, Song JD, Tian MM, Tian RG, Meng QL, Qiu F, Jia ZY and Bi SL: [The assemblage, purification and characterization of EV71 VLPs expressed in baculovirus]. Bing Du Xue Bao 28: 201-206, 2012 (In Chinese).

21. Jia CS, Liu JN, Li WB, Ma CM, Lin SZ, et al: The cross-reactivity of the enterovirus 71 to human brain tissue and identification of the cross-reactivity related fragments. Virol J 7: 47, 2010.

22. Meng T, Kolpe AB, Kiener TK, Chow VT and Kwang J: Display of VP1 on the surface of baculovirus and its immunogenicity against heterologous human enterovirus 71 strains in mice. PLoS One 6: e21757, 2011.

23. 21. Foo DG, Alonso S, Chow VT and Poh CL: Passive protection against lethal enterovirus 71 infection in newborn mice by neutralizing antibodies elicited by a synthetic peptide. Microbes Infect 9: 1299-1306, 2007.

24. Wang X, Peng W, Ren J, Hu Z, Xu J, Lou Z, Li X, Yin W, Shen X, Porta C, et al: A sensor-adaptor mechanism for enterovirus uncoating from structures of EV71. Nat Struct Mol Biol 19: 424-429, 2012.

25. Wen ZS, Xu YL, Zou XT and Xu ZR: Chitosan nanoparticles act as an adjuvant to promote both Th1 and Th2 immune responses induced by ovalbumin in mice. Mar Drugs 9: 1038-1055, 2011

26. Emeny RT, Wheeler CM, Jansen KU, Hunt WC, Fu TM, Smith JF, MacMullen S, Esser MT and Paliard X: Priming of human papillomavirus type 11-specific humoral and cellular immune responses in college-aged women with a virus-like particle vaccine. J Virol 76: 7832-7842, 2002.

27. Quan FS, Huang C, Compans RW and Kang SM: Virus-like particle vaccine induces protective immunity against homologous and heterologous strains of influenza virus. J Virol 81: 3514-3524, 2007.

28. Liu L, Zhao H, Zhang Y, Wang J, Che Y, Dong C, Zhang $\mathrm{X}, \mathrm{Na} \mathrm{R}$, Shi H, Jiang L, et al: Neonatal rhesus monkey is a potential animal model for studying pathogenesis of EV71 infection. Virology 412: 91-100, 2011.

29. Wang W, Duo J, Liu J, Ma C, Zhang L, Wei Q and Qin C: A mouse muscle-adapted enterovirus 71 strain with increased virulence in mice. Microbes Infect 13: 862-870, 2011.

30. Duo J, Wang W, Tong W, Cong Z, Jiang H, Xu W, et al: Experimental Studies on ICR Mice with EV71 Infection. Chin J Comp Med 19: 41-46, 2009.

31. Xiu JH, Zhu H, Xu YF, Liu JN, Xia XZ and Zhang LF: Necrotizing myositis causes restrictive hypoventilation in a mouse model for human enterovirus 71 infection. Virol J 10: $215,2013$. 